\section{Deutsche Gesellschaft für Hygiene und Mikrobiologie e.V.}

\author{
A. M. Gressner ${ }^{1}$ und O. A. Gressner ${ }^{2}$ \\ ${ }^{1}$ Labor Dr. Wisplinghoff Berlin, Berlin, Deutschland \\ ${ }^{2}$ Labor Dr.Wisplinghoff Köln, Köln, Deutschland
}

\section{Synonym(e) DGHM}

Englischer Begriff German Society of Hygiene and Microbiology

Definition Die DGHM ist eine wissenschaftliche Fachgesellschaft, die den Zusammenschluss aller in der Mikrobiologie und Hygiene tätigen Wissenschaftler in Deutschland anstrebt und das Fach auf den verschiedenen Teilgebieten in Forschung, Lehre und Krankenversorgung vertritt.

Beschreibung Die DGHM wurde im Jahr 1906 als „Freie Vereinigung für Mikrobiologie“ u. a. von P. Ehrlich ( $\triangleright$ Ehrlich, Paul), G. Gaffky, A.P. von Wassermann und R. Koch ( $\triangleright$ Koch, Robert) gegründet. Nach einer Umbenen- nung 1922 erhielt sie im Jahr 1949 ihre endgültige Bezeichnung „Deutsche Gesellschaft für Hygiene und Mikrobiologie e.V.“. Sie vertritt die Teilgebiete Mikrobiologie, Infektionsimmunologie, Hygiene und Gesundheitswesen in Forschung, Lehre und Krankenversorgung. Hierzu sind ständige Arbeitsgemeinschaften, Kommissionen und Fachgruppen eingerichtet. Die Förderung des wissenschaftlichen Austauschs auf Kongressen und sonstigen Tagungen stellt eine satzungsgemäße Aufgabe der DGHM dar.

\section{Adresse}

Deutsche Gesellschaft für Hygiene und Mikrobiologie e.V. c/o Medizinische Hochschule Hannover

Institut für Medizinische Mikrobiologie und Krankenhaushygiene

Carl-Neuberg-Str. 1

D-30625 Hannover

Tel.: 0511/5324655

Fax: 0511/5324355

E-Mail: dghm@mh-hannover.de

www.dghm.org 